\title{
La mitologizzazione del testo letterario: le Stanze del Poliziano
}

\author{
Attilio Manzi \\ Universidad de Sevilla
}

\begin{abstract}
Nelle Stanze del Poliziano, l'elemento mitologico è stato spesso considerato un vacuo accessorio della trama, un raffinato e stucchevole esercizio retorico fine a se stesso. Nell'articolo, si dimostra invece come esso risponda ad un progetto concreto dell'autore che elabora una riflessione sulla natura finzionale, sull'artificio letterario. Il discorso mitologico soppianta il discorso narrativo e apre al lettore un altro percorso di significati che hanno la loro origine nel gusto dell'epoca.
\end{abstract}

Parole chiave: mitologia, linguaggio parallelo, incipit metafinzionale, topos, percorso di lettura.

\section{Abstract}

In Poliziano's Stanze, the mythological aspect has often been seen merely as an adjunct to the plot, a refined and sterile rhetorical exercise. However, the article shows how it is part of a specific project by the author who reflects on the topic of fiction and literary artifice. The mythological discourse replaces the narrative discourse and opens up another semantic journey to the reader which has its origins in the taste of the era.

Key words: mythology, parallel language, incipit, topos, journey of reading.

Molto si è scritto, e molto ancora si dovrà aggiungere, a proposito del «poemetto» ${ }^{1}$ del Poliziano e, concretamente, riguardo alla sua evidente natura di testo eminentemente finzionale. Uno sguardo, seppure sbrigativo, alla non amplissima mole di letteratura critica accumulatasi nel corso di un secolo e mezzo, vale a dire da quando, col Carducci ${ }^{2}$ si inaugura la moderna riscoper-

1. Renzo Lo Cascio, Lettura del Poliziano. Le Stanze per la Giostra, Palermo: Flaccovio, 1954.

2. Giosuè CARDUCCI, "Delle poesie toscane di Angelo Poliziano", in Angelo POLIZIANO, Le Stanze, l'Orfeo e le Rime rivedute su i codici e su le antiche stampe e ilustrate con annotazioni di varii e nuove da Giosue Carducci, Firenze: Barbera, 1863. 
ta dell' opera, rivela sorprendentemente come la critica abbia tardato a riconoscere nel testo la sua natura di artificio di impianto finzionale. Come tale, esso ubbidisce a logiche e strategie costruttive specifiche, sinteticamente e provvisoriamente definibili come antimimetiche, in linea con quanto già affermato da Rolan Barthes: «La funzione del racconto non è di «rappresentare» ma è quella di costituire uno spettacolo che ci resta ancora assai enigmatico, ma che non può essere di ordine mimetico; la «realtà» d'una sequenza non risiede nella serie "naturale» delle azioni che la compongono, ma nella logica che vi si espone, vi si rischia e vi si compie». ${ }^{3}$ Stupisce invece constatare come, per lunghissimo tempo, gran parte della ricezione si sia concentrata sulla presunta vacuità (in termini, oggi ampiamente superati, di mancanza di "contenuto", «impegno», «ispirazione» ecc.), interpretando la sua ricercatezza formale (con il relativo apparato di immagini, suggestioni, citazioni) come mero decorativismo, dotto esercizio di stile presumibilmente vuoto di "messaggio». La ricercatezza formale delle Stanze, l'argomento trattato, l'apparente assenza dal testo di implicazioni ideologiche, morali, storiche, sociologiche, psicologiche, religiose e così via, sembrano infatti aver costituito per lungo tempo un elemento di difficoltà all' ora di collocare il testo in una giusta prospettiva critica. Il suo presentarsi come prodotto di finzione di natura fortemente estetica, ha spinto gran parte della critica tradizionale ad intendere la sua spiccata letterarietà come un elemento negativo. In questo senso ha esercitato un' influenza determinante la lettura offerta dal De Sanctis ${ }^{4}$ che è venuta ad inaugurare una tradizione a lungo vigente che, un po' sorprendentemente, non è riuscita, in sede di valutazione, a superare la dicotomia forma-contenuto, dove il termine «forma» veniva ad assumere connotazioni tendenzialmente negative. L'apparente sbilanciamento del testo polizianeo sul primo elemento, ha condotto a considerare spesso il livello stilistico-formale come semplice recipiente di un contenuto che, privilegiato da considerazioni di stampo storico ed ideologico, sembrava manifestamente assente. Solo letture abbastanza recenti come quella fondamentale (e scarsamente citata) di Ida Mäier, ${ }^{5}$ quella del Martelli ${ }^{6}$ e quelle nate dal rinnovato interesse per l'opera dovuto a Vittore Branca ed ai suoi discepoli, si sono avvicinate al testo da nuove prospettive «scoprendone» la natura pluridiscorsiva. Partendo da questo presupposto fondamentale, gli ultimi contributi critici si sono proposti di scandagliare singoli aspetti che ne costituiscono la letterarietà soffermandosi, per esempio, sul discorso filosofico-allegorico, su quello ermeneutico o quello dei legami intermediali che il testo stabilisce con testi contemporanei ma di altra natura artistica (fondamentalmente pittorica).

3. Roland BARTHES, «Introduzione all'analisi strutturale dei racconti» in L'analisi del racconto, Milano: Bompiani, 1969.

4. Francesco De SANCTIS, Storia della letteratura italiana, vol. I, Torino: Einaudi, 1971.

5. Ida MÄIER, Ange Politien: la formation d'un poète humaniste (1469-1480), Genève: Droz, 1969.

6. Mario Martelli, «Simbolo e struttura delle Stanze» in Stanze cominciate per la giostra di Giuliano de’ Medici, Alpignano: Tallone, 1979. 
Visto in quest' ottica plurale, il testo polizianeo si mostra dunque in tutta la propria complessità e si dimostra tutt'ora in grado di offrire un enorme potenziale al lettore interessato non tanto ad una critica delle fonti quanto ad un lavoro di scandaglio, di saggio e ricostruzione del patrimonio semantico del testo.

In effetti le Stanze, nel loro costituirsi in testo letterario, in struttura che si costruisce ed accresce progressivamente attraverso lo spazio testuale sulla scorta dell' impiego di molteplici strategie puramente finzionali, si presentano sin dalle primissime ottave come opera che allude, in modo nemmeno troppo nascosto, alla propria natura finzionale o, detto altrimenti, alla propria natura di artificio letterario. In questo senso sembra dunque opportuno sottoporre il testo ad un'analisi ispirata alle nuove teorie della fiction, identificando nel ricorso immediato ed insistito al discorso mitologico uno degli elementi chiave, uno dei "meccanismi di formalizzazione», direbbe Pavel, ${ }^{7}$ attuati da un testo che aspira a creare un universo finzionale. In questo senso appare particolarmente significativo il ricorso immediato ed insistito al discorso mitologico che è da interpretare, in via del tutto preliminare, quantomeno come elemento-chiave, rivelatore di una generale strategia autoriale che manifesta la propria presenza ed il proprio controllo sul testo, prefiggendosi programmaticamente di complicarne la semiosi sovrapponendo, sin dalle prime battute, al linguaggio messo al servizio dello sviluppo della diegesi, un linguaggio parallelo ed «altro», quello mitologico appunto. ${ }^{8} \mathrm{E}$ se non è questo il momento per procedere all' analisi del famoso incipit, troppe volte sbrigativamente ridotto alla funzione di obbligatoria introduzione di carattere retoricoesornativo-encomiastico, e mai letto come prologo-manifesto di natura decisamente metafinzionale, va pur detto come l'immediata presenza, sin dal quarto verso, di allusioni di tipo mitologico (nella fattispecie e in modo altamente significativo a Venere, "[di] quella dea che l' terzo ciel dipinge») deve far riflettere. Già Maria Luisa Doglio 9 ha avuto modo di procedere ad alcune interessanti considerazioni sul discorso mitologico delle Stanze rilevando appunto come il testo polizianeo sia sottoposto ad un forte processo di mitologizzazione che fa dell" opera una sorta di "puzzle di figurazioni e immagini simboliche che trovano nella mitologia una realtà che ha nel simbolo una sua ragion d'essere e il fine stesso della sua utilizzazione e non, come per Ficino, il punto di partenza di un' investigazione filosofica». ${ }^{10}$ Senza voler entrare in questa

7. Thomas G. PAVEL, Mondi di invenzione. Realtà ed immaginario narrativo, Torino: Einaudi, 1992.

8. Rimando a questo proposito alle note considerazioni fatte da Barthes nelle Mythologies, dove, per l'appunto, lo studioso analizza il mito partendo dalla sua condizione essenziale di linguaggio, vale a dire di sistema di significazione saussurianamente costituito da un significante ed un significato sorretti da un rapporto di reciproca motivazione che, nella loro interazione, danno luogo alla realtà tridimensionale del segno inteso come sistema semiologico che tende, per la sua stessa natura, ad una «ultrasignificazione».

9. Maria Luisa Doglio, «Metamorfosi, simbolo e favola. Per una lettura delle Stanze del Poliziano" in Italianistica, 12, p. 196-216.

10. Ibid., p. 215. 
sede nel merito di considerazioni che appaiono indubbiamente opportune, ${ }^{11}$ viene fatto comunque a questo punto di domandarsi quale sia la ragione di una presenza tanto consistente dell' elemento mitologico nelle Stanze.

Prima di procedere ad alcune considerazioni ${ }^{12}$ è forse utile riassumere brevemente il contenuto della storia, ricordando gli avvenimenti che la compongono al fine pratico di rilevare la presenza, a livello di organizzazione strutturale, di macrosequenze mitologiche.

Dopo il proemio e la dedica a Lorenzo il Magnifico, l'io lirico si rivolge ad Achille per chiedergli il permesso di interrompere la traduzione dell' Iliade a cui si stava dedicando per cantare le imprese guerriere ed amorose di Giuliano (qui latinizzato in Iulio). Il giovane, trascorre la sua gioventù cacciando con i propri amici ai quali confida il suo disprezzo nei confronti di chi non sa evitare le insidie d'amore. Cupido lo ascolta e, risentito, decide di vendicarsi mentre Iulio è impegnato in una battuta di caccia. Materializza dal nulla una cerva bianca che il cacciatore insegue nel fitto del bosco giungendo in una radura dove al posto della cerva gli appare una fanciulla di divina bellezza della quale Iulio, trafitto da Cupido, si innamora. Seduta sull' erba, ha intrecciato una ghirlanda di fiori e ne ha ancora pieno il grembo: dice di essere Simonetta Cattaneo e se ne va. Iulio torna a casa mentre Cupido, soddisfatto, vola al regno della madre, Venere, che il poeta, dopo aver invocato la musa Erato, descrive soffermandosi particolarmente sulla reggia e sui bassorilievi che ne ornano le porte. Qui, dove era nato, Cupido giunge e trova Venere nelle braccia di Marte con intorno degli amorini svolazzanti. Nel Libro secondo Cupido le racconta di avere fatto innamorare Iulio ed esalta la stirpe dei Medici. Venere decide che Iulio deve combattere nella giostra per rendere gloria ad Amore ed invia degli Amorini a Firenze affinché i giovani della città siano spinti a partecipare al torneo: così, mentre essi dormono, nei loro petti si accende un ardore guerriero e amoroso. Intanto Venere manda la più bella delle Grazie, Passitea, dal suo sposo il Sonno affinché, per mezzo dei sogni, induca Iulio a scendere in campo. Poco prima dell' alba il giovane ha una visione in cui Amore lo invita a rivolgersi verso la Gloria, la quale a sua volta lo riveste delle armi di Minerva e lo porta alla vittoria; ma la sua gioia si trasforma in lutto per il presagio della morte di Simonetta. La Fortuna si rivela quindi padrona dei destini umani e ad essa si può opporre soltanto la virtù.

11. Mi riferisco alla giusta presa di distanza da interpretazioni del testo polizianeo troppo rigidamente univoche volte a dichiararne la natura di esclusiva rappresentazione codificata del sistema teologico neoplatonico come proposto da Martelli che, seppur valide e senz' altro motivate, nel loro meccanicismo deterministico finiscono per privare il testo di buona parte della sua valenza suggestiva. Il mito, come si ricordava anteriormente citando Roland Barthes, è un sistema semiologico tendente per sua stessa natura all' ammissione di una varietà di significati.

12. La natura di questo contributo è volutamente preliminare e quindi generale rispetto ad un posteriore approfondimento della problematica che richiede un esaustivo lavoro di analisi e riscontro testuale. Anche per questo il riassunto schematico della fabula si limiterà a ricordare solo i nuclei essenziali attorno ai quali si organizza il discorso mitologico evitando di soffermarsi sulle loro molteplici derivazioni. 
Al suo risveglio Iulio invoca Minerva, la Gloria ed Amore promettendo di prendere parte alla giostra con un' insegna a loro ispirata.

Risulta evidente, da quanto esposto, come l'elemento mitologico, che viene introdotto in forma graduale nel corso della diegesi, poco a poco finisce col diventare una presenza non solo costante ma addirittura monopolizzante della poetica del testo. Il discorso mitologico, costruito attraverso un' accumulazione di materiale che procede in crescendo, finisce infatti per soppiantare il discorso strettamente narrativo, sovrapponendosi ad esso e imponendosi all'attenzione del lettore come presenza ineludibile. Il risultato è che il testo assume via via un carattere sempre più rarefatto: il passaggio in secondo piano dell'istanza narrativa implica automaticamente una complicazione della semiosi. Come ricorda Pavel ${ }^{13}$ la densità del testo a livello di trama diminuisce laddove aumentano i suoi percorsi epistemici. Il massiccio ricorso all'apparato mitologico rivela dunque al lettore il fatto che si trova di fronte ad uno scarto, ad un cambiamento di intenzioni e, di riflesso, dell'organizzazione testuale. Se, sempre con Pavel, ${ }^{14}$ il testo passa da una fase descrittiva retta dal mostrare (showing) ad una retta dal dimostrare (telling) ciò implica automaticamente la crescita della densità del testo che si fa più concentrato e più complesso. Nella fattispecie le Stanze, optano per una decisa complicazione della semiosi arricchendosi del codice mitologico che, col proprio apparato di immagini, topoi, allegorie ecc. propone l'esistenza di - per lo meno- un altro percorso epistemologico. Optando per questa variazione organizzativa e strutturale, sembra che l'autore dichiari implicitamente di aver esaurito il proprio interesse per un racconto di tipo impressionistico e, per contro, di voler procedere ad una variazione del paesaggio testuale: scompaiono elementi, personaggi, situazioni verosimili e, viceversa, fanno la loro comparsa elementi, personaggi e situazioni inverosimili. Naturalmente questa strategia di sovrapposizione o, meglio, di deviazione discorsiva implica un aumento del livello di esigenza del testo nei riguardi del proprio lettore che «guidato con mano ferma dall'autore, deve sottomettersi alla disciplina impostagli, per vedere ripagata la sua attenzione con un salto qualitativo nella comprensione del testo". ${ }^{15}$ In quest'ottica dunque il codice mitologico segna, col suo infittirsi, un radicale cambiamento di rotta: il testo «si affranca da ogni interesse referenziale» ${ }^{16}$ per avviarsi su un percorso di marcata tendenza allegorica. A partire da questo momento il mito diviene un filtro attraverso il quale sembra che debba obbligatoriamente passare la fruizione del testo. L'insistito inserimento di figure mitologiche, a formare metafore ed allegorie, rivela dunque l'esistenza di un preciso progetto estetico, l'esistenza di una precisa volontà significante occulta sotto quello che, per molto tempo, è sembrato solo uno stucchevole gioco letterario. Qual è dunque il senso di questo deciso processo di mitologizzazione a cui

13. Thomas G. PAVEL, op. cit., p. 139.

14. Ibid., p. 153.

15. Ibid., p. 154.

16. Ibid., p. 154. 
viene sottoposta la materia narrativa? Ed ancora, che significato assume questo processo di codificazione, questa evidente volontà di adottare un metalinguaggio elaborato sulla base del materiale mitologico?

Si potrebbe cominciare con una considerazione di carattere generico: la mitologizzazione del testo artistico risponde innanzitutto a ragioni di ordine estetico in generale $\mathrm{e}$, più concretamente, a ragioni di gusto. E quando parlo di gusto lo faccio con la piena coscienza di usare un concetto a cui studiosi della storia dell' arte, soprattutto dopo il fondamentale contributo di Francis Haskell, ${ }^{17}$ hanno riconosciuto da tempo dignità di categoria critica ma che, nell'ambito della critica letteraria, sembra che desti tutt'ora certa diffidenza. In ogni caso, in questo senso mitologizzare un testo artistico equivarrebbe ad attualizzarlo in conformità col canone estetico vigente all' epoca della sua produzione. Per quanto riguarda le Stanze, ciò significa inserire il testo letterario all' interno di un più ampio contesto culturale in cui le idee filosofiche ed artistiche circolano e si frammischiano con estrema facilità grazie soprattutto alla presenza di un centro di potere che si propone come punto di coordinazione e diffusione delle stesse. La sodalitas di artisti di varie discipline attorno ad un progetto esteticamente omogeneo finisce così per impregnare di sè tutta la produzione artistica contemporanea a Poliziano. E tra la vastissima letteratura che ha approfondito questo tema basti solo ricordare i notissimi studi di Panovsky, ${ }^{18}$ Gombrich, ${ }^{19}$ Wind ${ }^{20}$ e Kristeller. ${ }^{21}$

Ricorrere ad un esteso uso di figure mitologiche avrebbe dunque il significato di corrispondere ad una precisa domanda proveniente dal pubblico lettore e dal committente che richiedono entrambi un prodotto in linea con l'estetica dominante. Il gusto per l'elemento mitologico va dunque letto da un lato, come uno dei momenti agglutinanti, uno dei leitmotiv di tutta la produzione artistica contemporanea al concepimento delle Stanze e, dall' altro, come esplicante una funzione estetica o, per dirla con Lotman, «rafforzativa [...] della crescita del significato estetico, che nella narrazione artistica svolgeva prima un ruolo solo subalterno rispetto alla funzione sacrale o a quella pratica» (208). ${ }^{22}$ Mitologizzare un testo poetico corrisponde dunque ad una esigenza artistica in senso lato: il mito compie una funzione aumentativa, amplificativa della capacità estetica del testo e, parallelamente, della possibilità di fruizione, estetica appunto, da parte del suo lettore.

Evidentemente, una volta riconosciuta la funzione estetizzante del mito bisogna andare oltre, in quanto, per citare sempre Lotman, «ogni oggetto culturale può funzionare soltanto se il suo meccanismo ha le proprietà dell' ete-

17. Francis Haskell, Riscoperte nell' arte, Milano: Edizioni Comunità, 1990.

18. Erwin PANOFSKY, Rinascimento e rinascenze nell' arte occidentale, Milano: Feltrinelli, 1971.

19. Ernst Hans GOMBrICH, "Mitologie botticelliane» in Immagini simboliche. Studi sull' arte del Rinascimento, Torino: Einaudi, 1978, p. 47-113.

20. Edgar WIND, I misteri pagani del Rinascimento, Milano: Adelphi, 1971.

21. Paul Oskar KRISTELleR, Il pensiero e le arti nel Rinascimento, Roma: Donzelli, 1998.

22. Juri M. LOTMAN, La semiosfera. L'asimmetria ed il dialogo nelle strutture pensanti, Venezia: Marsilio, 1992. 
rogeneità e del poliglottismo semiotico». ${ }^{23}$ Procedendo sempre da osservazioni preliminari ad altre più sofisticate, si può riconoscere allora nell' intensa mitologizzazione delle Stanze anche una funzione encomiastica. Parte del materiale mitologico che compare nel poema infatti viene messo al servizio del discorso encomiastico-celebrativo che attraversa l'opera. Scritte per cantare il trionfo di Giuliano de' Medici nella giostra celebrata a Firenze il 29 gennaio 1475, le Stanze riprendono la tradizione inaugurata sei anni prima da Luigi Pulci che aveva cantato il successo di Lorenzo il Magnifico in una giostra simile. La giostra, da momento di legittimazione ed esibizione del potere acquisito diventa, a livello testuale, motivo generatore del discorso encomiastico che si manifesta attraverso la precisa combinatoria di frammenti mitologici. Vi è un passaggio, verso la fine del secondo libro, dove quest' intenzionalità celebrativa sottesa all' uso del materiale mitologico diviene particolarmente manifesta: la descrizione del sogno di Iulio (II, 28). A Iulio appare in sogno la visione di Amore sconfitto e reso prigioniero da Simonetta rappresentata nelle vesti di una Minerva guerriera, spietata ed insensibile, vestita con una gonna bianca, con in mano uno scudo rappresentante la testa di Medusa. Essa lo invita ad ispirarsi alla Gloria per armarsi ed intervenire a difenderla. Di seguito la Gloria scende - significativamente accompagnata dalla Poesia e dalla Storia - e veste Iulio con la corazza, lo scudo e le armi tolti a Simonetta e la Fortuna lo guida al conseguimento della fama eterna. A questo punto Iulio si sveglia ed invoca Amore, Minerva e la Gloria affinché lo conducano alla vittoria. All' interno dello spazio circolare del «fallace sonno» (II, 30) viene così tracciato un insieme di coordinate simboliche che vanno dal topos della Fortuna capricciosa a Minerva che lega Cupido al tronco dell' ulivo a lei sacro, al mito della testa di Medusa che poteva pietrificare con lo sguardo e che fu recisa da Perseo e poi fissata sullo scudo di Minerva. Il tutto a proiettare il protagonista direttamente al centro del mito. Rappresentato come un guerriero classico (con tutti gli attributi anche psicologici che lo caratterizzano), Iulio si prepara al combattimento - anche se solo in sogno- indossando l'armatura della dea ed impossessandosi in tal modo dei suoi atributi che, difatto, esibisce. La stessa scena, tra l' altro, riprende poi esattamente ciò che rappresentava nella realtà storica lo stendardo dipinto da Botticelli che portava il vessillifero di Giuliano il giorno del torneo attivando uno degli innumerevoli intertesti figurativi che attraversano il poema e, ripeto, sottolineando una volta di più attraverso l'esibizione di un materiale mitologico appositamente scelto, la condizione d'eccellenza del destinatario dell' encomio. Il materiale scelto a proposito è estremamente efficace. La divinità principale chiamata in causa è la maestosa Minerva che, oltre ai noti attributi guerrieri (nasce dalla testa di Giove completamente armata) riunisce una serie di attributi intellettuali di prim' ordine: è infatti simbolo dell' intelligenza, della saggezza e delle arti e protettrice della pace. Chi avrebbe potuto assistere meglio l'eroe nelle sue imprese non solo strettamente sportive ed amorose, ma di ampio significato politico? 
Tuttavia tanto la funzione estetica quanto quella encomiastica svolte dal mito nelle Stanze sono secondarie rispetto a quella principale, quella che potremmo definire come metadiscorsiva. L'insistito accumularsi del materiale mitologico lungo l'itinerario disegnato nell'opera, informa il lettore dell' esistenza di un discorso codificato che trascorre parallelo allo sviluppo della fabula. Dietro le immagini create dal materiale mitologico selezionato dall' autore è lecito immaginare che si nasconda qualcosa di più. Se le figure mitologiche nascono come veicoli di una significazione "altra», come strumenti di trasmissione di contenuti codificati, il loro impiego intenso da parte di Poliziano autorizza —anzi, obbliga - il lettore a ricercare dietro di esse o dentro di esse l'esistenza di un discorso che si sviluppa parallelamente a quello strettamente narrativo e che per essere compreso deve essere previamente decodificato. Il materiale mitologico è dunque impiegato come recettore di un discorso in codice che l'autore consegna al suo lettore. E per la sua natura di linguaggio cifrato sarà proprio in esso, nella decifrazione della combinatoria - tutt'altro che fissa e prevedibile- delle allegorie e dei simbolismi, dove bisognerà ricercare uno dei principali generatori di senso dell' opera. Il materiale mitologico organizzato in topoi svolge dunque la funzione di "collettore della semiosi e di suo momento disciplinante»: ${ }^{24}$ esso serve per «orientare la direzione delle attualizzazioni previste dalla strategia approntata dall' autore». ${ }^{25}$ Detto altrimenti: il codice mitologico è un veicolo, agisce come una chiave che dà l'accesso al mondo finzionale che, a partire dal superamento di questa sorta di soglia, si apre ed espande. Un'espansione che, come ricorda Pavel, è condannata ad un andamento irregolare ed imprevisibile, visto che «dal momento che i mondi reali appaiono veri, completi e coerenti al di là di ogni dubbio [...] i mondi di invenzione sono intrinsecamente incompleti e incoerenti». ${ }^{26}$

Ritornando ancora una volta allo schema narrativo delle Stanze non sarà difficile dunque riconoscere come l'innesto dell' elemento mitologico stimola il lettore ad avventurarsi sulla via di un percorso di lettura che si svolge ad un diverso livello testuale. L'inattesa apparizione di una ninfa dalle sembianze di Simonetta Cattaneo al posto della cerva bianca che Iulio stava inseguendo, lo induce — «tutto ripien di maraviglia» (I, 38) — a pensare che sta succedendo qualcosa di strano e contemporaneamente mette sull' avviso lo stesso lettore che quest' intervento divino postula l'esistenza di un altro possibile percorso di lettura. L'apparizione della ninfa rompe l'ordinato succedersi degli avvenimenti della sequenza narrativa e crea un' attesa: è ciò che Eco definisce un segnale di suspense vale a dire un "segnale testuale» introdotto per sottolineare che la disgiunzione che sta per occorrere è rilevante. [...] entrare in stato di attesa significa far previsioni. Il lettore Modello è chiamato a collaborare allo sviluppo della fabula anticipandone gli stati successivi. [...] nel fare queste previsioni il lettore assume un atteggiamento proposizionale [...] circa il modo

24. Umberto ECO, Lector in fabula, Milano: Bompiani, 1979, p. 89.

25. Ibid., p. 89.

26. Thomas G. PAVEL, op. cit., p. 112. 
in cui andranno le cose. Così facendo configura un possibile corso di eventi o un possibile stato di cose: azzarda ipotesi su strutture di mondi [...] possibili. ${ }^{27} \mathrm{Ma}$ questo segnale è altresì pretesto affinché il lettore Modello faccia una passeggiata inferenziale, fuoriesca dal testo attarverso il topos che è chiamato ad attuare per rientrarvi "carico di bottino intertestuale».

Il topos dell' apparizione della cerva è dunque il momento generatore di un livello narrativo più profondo la cui chiave di accesso è data dalla decifrazione del codice mitologico che lo descrive. In questo senso Poliziano usa il materiale mitologico della tradizione classica sottoponendolo ad un processo di risemantizzazione. Ma proprio la natura aperta del codice mitologico ha favorito varie possibilità di lettura, ha aperto vari percorsi ermeneutici principalmente volti a risaltare la tendenza del testo ad espandersi in una direzione metafisico-filosofica. Vittore Branca, per esempio, recuperando una delle prime letture delle Stanze che ne evidenziano la natura simbolico-allegorica, quella già ricordata di Mario Martelli, ha identificato un «itinerario ficiniano» ${ }^{28}$ costruito attorno alla figura centrale di Venere. In essa l'autore ha sottolineato come, sulla scorta di testi filosofici di Marsilio Ficino e di altri autori del neoplatonismo fiorentino, Poliziano costruisca una grande allegoria: «il cervo, detentore presso la tradizione di molteplici significati, simboleggia [...] il «desiderium salvantis», l'intenso desiderio che il Salvatore ha di riscattare l'anima umana da lui amata [...]; da questo si potrà sicuramente dedurre che appunto per l'intervento della divina misericordia, Iulio viene avviato alla difficile strada della perfezione che, dalla cerva dei sensi, lo conduce alla Simonetta delle virtù civili: mentre in prospettiva si delinea il regno di Venere che, trasposizione classica del cristiano paradiso terrestre, simboleggia la forma più alta di vita, quella contemplativa». ${ }^{29}$

Il materiale mitologico, secondo questa possibile, plausibile ma non unica interpretazione, viene dunque svuotato del contenuto consegnato dalla tradizione classica per divenire ricettore di un discorso filosofico di matrice neoplatonica che, a sua volta, sconfina in un più ampio discorso religioso: teologia platonica e teologia cristiana si sovrappongono e fondono all' interno di un unico «continente». In questo procedere per contaminazioni consecutive, per costanti sovrapposizioni ed interpolazioni di significato, a formare un tessuto discorsivo estremamente stratificato, va riscontrata l'essenza stessa dell' estetica del Rinascimento fiorentino. "Simonetta si presenta a Iulio come figlia di Venere e, perciò, come amore che l'anima ha della bellezza divina - amore celeste, per questo, ma non perfetto, bensì immagine perfetta e propinqua di quello. In altre parole, esso sarà l'amore delle virtù terrene (civili e politiche, cioè) che, nella scala ficiniana, si sostituisce a quello pandemio o volgare. L'uomo che gode di questo amore ha, secondo Ficino (e poi secondo Pico), raggiunto il secondo gradino della scala d'amore, donde potrà ascendere al terzo, della 
bellezza angelica, per poi (ma solo dopo la morte del corpo) innalzarsi al quarto, che è quello della bellezza divina». ${ }^{30}$ Questo, nell' interpretazione di Martelli, vale a dire nella sua personale discesa all' interno del multiforme mondo di significato motivato dalla capacità allusiva di una figura mitologica di particolare efficacia.

Tuttavia, ciò che interessa in questa sede non è tanto procedere a interpretazioni esaustive quanto segnalare come il materiale mitologico svolga la funzione principale di segnare il confine tra due diversi mondi all'interno del testo letterario; uno, che si potrebbe definire, sempre con Pavel, "primario» e l'altro "secondario", entrambi caratterizzati da strutture ontologiche diverse. Il «mondo secondario» a cui si accede attraverso il mito è un mondo di invenzione retto da peculiari strutture, logiche e scopi tanto letterari quanto estetici che vanno innanzitutto riconosciuti in quanto tali e, successivamente, analizzati partendo dalla loro intrinseca e necessaria vocazione alla complessità, all'instabilità ed alla indeterminatezza.

Attraverso il codice mitologico il testo non solo manifesta la propria natura pluridiscorsiva quanto soprattutto tematizza la propria volontà estetica e la propria ricercatissima letterarietà. Il mito, per la sua natura allegorica, costituisce una delle strategie fondamentali di cui l'autore dispone per costruire un mondo di finzione che, in quanto tale, si nutre di letterarietà. È un elemento ludico fondamentale che, per la propria natura «ospitale» ed aperta, si dimostra naturalmente propenso a offrire tanto all'autore come al lettore la possibilià di imboccare costantemente nuovi itinerari interpretativi, nuovi percorsi di senso che allargano irrimediabilmente la semiosi del testo. In quest' ottica il repertorio di topoi apparentemente noti che prodigano la loro presenza nell' arco della narrazione, propongono con insistenza la propria vocazione emblematica, si offrono al lettore con fare interrogativo circa il senso della propria presenza. Ed è forse questa la loro principale ragion d'essere come elemento significante all' interno di qualsiasi testo artistico: quella di rappresentare con la propria ambiguità e duttilità il veicolo privilegiato dell' espressione poetica che trova il suo spazio d'elezione nel non detto, nel suggerito, nell' allusione a codici di riferimento vicini o lontanissimi, noti o tutti da scoprire. 